\title{
Experimental Comparison of Parametric Versus Nonparametric Analyses of Data From the Cold Pressor Test
}

\section{Citation}

Treister, Roi, Christopher S. Nielsen, Audun Stubhaug, John T. Farrar, Dorit Pud, Shlomo Sawilowsky, and Anne Louise Oaklander. 2015. "Experimental Comparison of Parametric Versus Nonparametric Analyses of Data From the Cold Pressor Test." The Journal of Pain 16 (6) (June): 537-548. doi:10.1016/j.jpain.2015.03.001.

\section{Published Version}

10.1016/j.jpain.2015.03.001

\section{Permanent link}

http://nrs.harvard.edu/urn-3:HUL.InstRepos:34627552

\section{Terms of Use}

This article was downloaded from Harvard University's DASH repository, and is made available under the terms and conditions applicable to Open Access Policy Articles, as set forth at http:// nrs.harvard.edu/urn-3:HUL.InstRepos:dash.current.terms-of-use\#OAP

\section{Share Your Story}

The Harvard community has made this article openly available.

Please share how this access benefits you. Submit a story.

Accessibility 
Published in final edited form as:

J Pain. 2015 June ; 16(6): 537-548. doi:10.1016/j.jpain.2015.03.001.

\title{
Experimental comparison of parametric versus nonparametric analyses of data from the cold pressor test
}

\author{
Roi Treister ${ }^{a, b}$, Christopher S. Nielsen ${ }^{c}$, Audun Stubhaug ${ }^{d}$, John T. Farrare, Dorit Pud ${ }^{f}$, \\ Shlomo Sawilowsky ${ }^{g}$, and Anne Louise Oaklander ${ }^{a, b, h}$ \\ aDepartment of Neurology, Massachusetts General Hospital, Boston, MA, USA \\ bHarvard Medical School, Boston, MA, USA \\ 'Division of Mental Health, Norwegian Institute of Public Health, Norway \\ dDepartment of Pain Management and Research, Oslo University Hospital, Norway \\ eDepartment of Biostatistics and Epidemiology, Perelman School of Medicine, University of \\ Pennsylvania, Philadelphia, PA, USA \\ ${ }^{f}$ Faculty of Social Welfare and Health Sciences, University of Haifa, Israel.ci d H \\ ${ }^{9}$ College of Education, Wayne State University, Detroit, MI, USA \\ hDepartment of Pathology (Neuropathology), Massachusetts General Hospital, Boston, MA, USA
}

\section{Abstract}

\begin{abstract}
Parametric statistical methods are common in human pain research. They require normally distributed data, but this assumption is rarely tested. The current study analyzes the appropriateness of parametric testing for outcomes from the cold pressor test (CPT), a common human experimental-pain test. We systematically reviewed published CPT studies to quantify how often researchers test for normality and how often they use parametric vs. non-parametric tests. We then measured the normality of CPT data from 7 independent small-to-medium cohorts and one study of $>10,000$ subjects. We then examined the ability of two common mathematical transformations to normalize our skewed data-sets. Lastly, we performed Monte Carlo simulations on a representative dataset to compare the statistical power of the parametric t-test vs. the nonparametric Wilcoxon Mann Whitney (WMW) test. We found that only 39\% of published CPT studies (47/122) mentioned checking data distribution, yet 72\% (88/122) used parametric statistics. Furthermore, among our 8 data sets, CPT outcomes were virtually always non-normally distributed and mathematical transformations were largely ineffective in normalizing them. The simulations demonstrated that the non-parametric WMW test had greater statistical power than the
\end{abstract}

(c) 2015 Published by The American Pain Society.

Corresponding author: Roi Treister, Ph.D., Massachusetts General Hospital, 275 Charles St./Warren Bldg. 310, Boston, MA 02114. Tel: 617-726-7632, Fax: 617-726-6991, treister.roi@ gmail.com.

Disclosures:. None of the authors have conflicts of interest.

Publisher's Disclaimer: This is a PDF file of an unedited manuscript that has been accepted for publication. As a service to our customers we are providing this early version of the manuscript. The manuscript will undergo copyediting, typesetting, and review of the resulting proof before it is published in its final citable form. Please note that during the production process errors may be discovered which could affect the content, and all legal disclaimers that apply to the journal pertain. 
parametric t-test for all scenarios tested-for small effect sizes, the WMW had up to $300 \%$ more power.

\section{Keywords}

Non parametric analysis; Wilcoxon Mann Whitney test; t-test; Cold Pressor Test; Tolerance

\section{Introduction}

Pain research is challenging because of the subjective nature of sensation, but during the last two decades pain research in humans has advanced dramatically due in part to the development and validation of multiple outcome measures. ${ }^{6,31}$ However, the statistical methods used to determine if differences in outcomes between two study groups are or are not significant have not been subject to rigorous evaluation. Even in well-designed and conducted studies, using the wrong statistical test to analyze outcomes can have major implications for the study's power. Erroneous conclusions can derail the progress of a promising new pain treatment, or slow the pace of understanding pain mechanisms.

This study evaluates the prevalence and appropriateness of parametric vs. nonparametric statistical analyses in evaluating measured differences in the perception and severity of pain sensations between two groups of subjects. A fundamental principle of statistics is that parametric analyses such as the t-test and analysis of variance (ANOVA) are only accurate if the data being analyzed comes from a population in whom the outcome variable is normally distributed ${ }^{24}$ Hence, researchers are supposed to check that their data are normally distributed before applying parametric analyses.

Contrary to the impressions of statistical pioneers such as Gauss and Galton, ${ }^{14}$ the normality of real-world distributions cannot be taken for granted. The largest study of psychometric and education measures found that none of the 440 data sets studied were normally distributed and only $3 \%$ remotely resembled a normal curve (i.e., symmetric with light tails). ${ }^{14}$ Studies from medical fields have generated similar conclusions. Perhaps closest to pain research is Colliver et al. study of in 68 cohorts comprising 1,326 patients undergoing general anesthesia where neither blood $\mathrm{pH}$ nor $\mathrm{H}^{+}$measurements were normally distributed. ${ }^{5}$ However, distribution of the outcome data is often not mentioned in published manuscripts. ${ }^{22}$ Insofar as we know, only two previous pain related studies have compared the use of parametric vs. nonparametric analysis. ${ }^{33,27}$ Here we focus on the cold pressor test (CPT), a widely used human-pain experimental paradigm. ${ }^{12,15,34}$ In the CPT, subjects are asked to immerse one hand in a cold water bath and to keep it submerged for as long as possible up to a safety limit (normally $1-5$ minutes). Three outcomes are commonly measured: Subjects indicate the time when the cold becomes painful ("cold pain threshold"), and when it becomes so painful that they must withdraw their hands from the water ("cold pain tolerance"). Subjects also rate their maximal pain intensity during immersion on a numerical pain scale at the end of the procedure ("cold pain intensity"). ${ }^{15}$ Although developed for other purposes, the CPT is commonly used in experimental pain 
research because it is simple to administer, reliable, inexpensive, and induces strong pain sensations.

In the current paper, we examined the consequences of applying parametric and nonparametric analyses to these three measures in several ways. First we conducted a systematic critical review of published CPT studies to define how often data normality was examined, the use of transformations and whether parametric or non-parametric analyses had been used. Then we examined the normality of multiple sets of our own CPT data to assess how often real CPT data in normally distributed and we applied corrective mathematical transformations to evaluate the success of this procedure to normalize the data. Lastly, we performed a Monte Carlo simulation study ${ }^{13}$, based on the CPT datasets to compare the power of the parametric t-test against the non-parametric Wilcoxon Mann Whitney test (WMW).

\section{Materials and Methods}

\section{Characterizing the statistical approaches used in published CPT studies}

We searched the MEDLINE database on March $1^{\text {st }} 2014$ using the terms: 'cold pressor test' AND 'tolerance' AND 'pain'. This identified 193 articles, of which we were able to obtain the full text from 141. Among them, 8 were excluded because cold-pain outcomes were not the dependent variable, 9 because cold-pain tolerance was not assessed using time to hand withdrawal, and 2 were reviews. Ultimately, 122 articles were analyzed.

Supplemental Table 1 in the Appendix summarizes the parameters we collected from each study: (1) PMID number (2) country of origin, (3) year of publication, (4) statistical approach (parametric versus non-parametric), (5) mention of the normality of the distribution (any mention, regardless of outcome), (6) use of mathematical transformations. Additional data collected but not shown in Table S1 included journal name, first author, number of subjects, group allocation (balanced or not), study design (between/within subject analysis), P-value, effect size (calculated, when possible), and approach for handling nonnormal distribution (if applicable and available). When assessing study design (between/ within subject analysis), studies were categorized as a "between-subject" analysis if cold pain tolerance was compared between groups at least once. We defined "balanced groups" as samples that differed in size ( $\mathrm{n}$ value) by no more than $30 \%$. In case inexact significant $\mathrm{P}$ values (i.e. $\mathrm{P}<0.05, \mathrm{P}<0.01, \mathrm{P}<0.001)$ were reported $(\mathrm{n}=37)$, or when non-significant $\mathrm{P}-$ values were not reported (i.e. 'none significant' was reported but with no P-value; n=20) Pvalues were coded as missing data.. Forty four studies reported their mean values and standard deviations or standard errors and these used to calculate the effect sizes. In studies making multiple comparisons, the largest effect size was recorded.

\section{Analysis of outcome data from different independent CPT studies}

CPT data were analyzed from 648 healthy volunteers who served as subjects in 7 independent pain research studies performed in the Human Experimental Pain Research Laboratory at the University of Haifa, Israel. All studies were conducted in the same laboratory, using the same methods and apparatus, and all subjects provided written 
informed consent to a research protocol that had been approved by the university ethical committee. All subjects were student volunteers recruited through advertisements posted at local universities. Across all studies, subjects were eligible for enrollment if they were healthy and free from chronic pain, did not use medications other than oral contraceptives, and could understand the study's purpose and instructions. Exclusion criteria were any type of medical or painful condition, pregnancy, use of medications, or history of drug abuse.

Haifa studies \#1 ( $n=40$, Pud 2010, unpublished data) and \#6 $\left(n=109^{18}\right)$ had assessed the role of hand dominance in pain sensitivity; study \#2 ( $n=48$, Pud 2009, unpublished data) had studied the association between experimental pain outcomes and sleep-related measures; study \#3 ( $n=62$, unpublished data) had assessed the effects of virtual reality on experimental pain outcomes; study \#4 $\left(\mathrm{n}=91^{17}\right)$ had assessed the associations between experimental pain outcomes and personality dimensions; study \#5 $\left(n=105^{28}\right)$ had assessed the effects of apomorphine on pain sensitivity; and study \#7 $\left(n=193^{29}\right)$ had assessed the association between experimental pain and candidate genes. In cases where the study had assessed the effect of an intervention (studies \#3 and \#5), the baseline measures (before intervention) were analyzed.

The Heto CBN 8-30 Lab cold pressor apparatus (Allerod, Denmark) was used for all Haifa studies. This temperature-controlled water bath is continuously stirred by a pump. Subjects placed their right hands with fingers spread into the water bath, whose temperature was maintained at $1{ }^{\circ} \mathrm{C}$. A stopwatch measured the thresholds of cold pain and cold pain tolerance. A 180 s cut-off time was used for safety, and the pain tolerance for subjects who did not withdraw their hands for the entire $180 \mathrm{~s}$ was recorded as $180 \mathrm{~s}$. immediately after hand withdrawal, subjects verbally rated their maximal pain intensity using a $0-100$ numerical pain score (NPS).

To evaluate very large datasets, we also analyzed CPT data collected at Troms $\emptyset$ University, Norway. Data were available from 10,486 individuals, $51.5 \%$ women, age 30-87 years. Participants had been recruited from the general population of the Troms $\varnothing$ municipality in northern Norway (for details see ${ }^{11}$ ). All subjects had provided written informed consent to a protocol approved by the regional ethical committee. The response rate was $65.7 \%$. Participants had been excluded from CPT if they reported previous health problems in connection with cold-exposure (e.g. cold-allergy, Reynaud's phenomenon) or if they were unable understand instructions. No other exclusion criteria were applied.

The Troms $\varnothing$ apparatus consisted of a refrigerated water bath FP40HE (Julabo Lobortechnik $\mathrm{GmbH}$, Germany) connected to an external 13L Plexiglas container. Water had been circulated at $13 \mathrm{~L} / \mathrm{min}$ to maintain water temperature at $3{ }^{\circ} \mathrm{C}$ as measured in the external container. Participants had placed their dominant hand and wrist in the water and maintained it there as long as possible, up to a maximum of $106 \mathrm{~s}$. Numeric pain ratings (0-10) had been obtained at 4 seconds and every 9 seconds thereafter until hand withdrawal; the coldpain tolerance time had been recorded by a stopwatch.

Although visual inspection of the data histograms clearly demonstrated non-normal distributions, statistical assessment of normality was additionally performed for descriptive 
reasons using the SPSS for Windows version 19 statistical package (SPSS, Inc., Chicago, IL). The Shapiro-Wilk test (SW) and Kolmogorov-Smirnov tests (KS) were used to assess the normality of raw and the transformed data. Data was judged to be normally distributed if $\mathrm{p}>0.05$ on either the SW or KS tests.

\section{Monte Carlo simulations for comparing statistical power of the Wilcoxon Mann Whitney test (WMW) and the t-test in assessing CPT outcomes}

We performed Monte Carlo simulations using all three CPT outcomes (intensity, tolerance, and threshold) obtained from all cohorts. Simulation results based on the baseline measures of dataset number 5 are described in detail. To demonstrate that these results are not cohort specific, examples of simulation results from the Troms $\emptyset(n=10,486)$, smallest Haifa (cohort $\# 1, n=40$ ), and the largest (cohort \#7, n=193) cohorts are described.

A program was written for R (64 bit version 3.02 (2013-09-25). The CPT dataset was sampled with replacement. The independent-samples t-test and the WMW test were conducted assuming homoscedasticity. The number of repetitions per experiment was $1,000,000$. Nominal a was set at $0.05,0.01$, and 0.001 . For each CPT outcome, 16 simulations with different $\mathrm{n}$ scenarios were conducted as follows: $\left(\mathrm{n}_{1}, \mathrm{n}_{2}\right)=(20,20),(15,25)$, $(25,15),(30,30),(15,45),(45,15),(45,45),(60,30),(30,60),(60,60),(45,90),(90,45)$, $(120,120),(150,90),(90,150)$, and $(240,240) . \mathrm{n}_{1}$ and $\mathrm{n}_{2}$ values were randomly sampled with replacement from the data set. To model a "difference in location" parameter, a specific effect size (i.e., fraction or multiple of the data set's standard deviation) was added to $\mathrm{n}_{2}$ for each iteration of an experiment. The added effect sizes to model a treatment in shift in location parameters were $\mathrm{d}=0.2 \sigma, 0.5 \sigma$, and $0.8 \sigma$, which Cohen defined as small, medium and large, respectively ${ }^{4}$; and $1.2 \sigma$ and $2.0 \sigma$, defined by Sawilowsky as very large and huge, respectively, where $\sigma$ defined as the population standard deviation of data set $\# 5^{23}$. One- and two-tailed Type I error rates of the WMW and the t-test are presented below:

One Tailed Test-The t-test's Type I errors for data sampled from the intensity and tolerance datasets were within sampling error of nominal a. However, the t-test's Type I errors for data sampled from the threshold dataset for nominal $\alpha=0.05$ was .0470 , for $a=$ 0.01 was .0075 , and for $a=0.001$ was .0005 . Although these results met Bradley's $(1978)^{2}$ liberal definition of robustness at $0.5 a \leq$ Type I error $\leq 1.5 a$, they did not meet Bradley's conservative definition of robustness at $0.9 \mathrm{a} \leq$ Type I error $\leq 1.1 \mathrm{a}$. The WMW test, being nonparametric, produced correct Type I errors within sampling errors.

Two Tailed Test-Type I errors for data sampled from the intensity and tolerance datasets for the non-directional layout also yielded results within the sampling error of nominal $a$. The t-tests results for the threshold data, however, were non-robust. With nominal $\alpha=0.05$, the expected Type I error rates are 0.025 at each tail, but the obtained rejection rates were conservative at 0.0208 and 0.0230 , respectively. Similarly, the lower tail rejection rates for nominal $\mathrm{a}=0.01$ (0.005 expected) and .001 (0.0005 expected) were .0069 and .0002 , respectively. 


\section{Results}

\section{Assessment of statistical approach used in published CPT studies}

The 122 articles analyzed (see Table S1 in the appendix) were published between 1977 and 2013. The mean number of subjects per study was $71.9 \pm 80$ (range $=4-613$, with plus articles with $\mathrm{n}>10,000$ excluded to avoid enlarging the mean). The mean reported P-value was $0.0516 \pm 0.12$ (range $\mathrm{P}=0.001$ to $\mathrm{P}=0.61$ ). $17.2 \%$ (21) of studies reported nonsignificant differences. We were able to calculate effect sizes for 44 studies and among them the mean effect size was $0.69 \pm 0.54$ (range 0.04 to 2.89 ). In $72 \%$ effect sizes were less than 0.8 , meaning small or moderate.

Overall, $72.1 \%$ of the studies (88/122) used parametric analyses and $27.9 \%(34 / 122)$ used non-parametric analyses. $71.3 \%$ (87) used between-subjects comparisons and among them, $74.7 \%$ (65) used parametric analyses and $25.3 \%$ used non-parametric analyses. Group sizes were balanced in $77.9 \%$ of studies and unbalanced in $22.1 \%$. In $61.5 \%$ of the studies (75) no information about normality of the data distribution was provided. Among the $38.5 \%$ of studies that mentioned testing for normality, $82.1 \%$ found that cold-pain tolerance was nonnormally distributed; among them $44.2 \%$ used logarithmic (34.1\%) or other (10.1\%) transformations to try and improve the normality of their datasets, and $37.9 \%$ used nonparametric analyses.

One cannot conclude that a given study used the appropriate parametric/non-parametric analysis without inspection of its raw data distribution. However, we can conclude that $37 \%$ (45/122) of the reviewed study's conclusions might be at risk since in these studies: (1) data normality was not tested; (2) between subject design was used and (3) parametric analysis was applied.

\section{Outcome data from different independent CPT studies}

Table 1 summarizes the analyses of the 7 Haifa cohorts plus all the Haifa cohorts combined $(n=648) .95 .8 \%(23 / 24)$ of the CPT outcomes were non-normal with only cold-pain threshold in the smallest cohort (\#1) being normally distributed based on the Shapiro-Wilk test but not on the Kolmogorov-Smirnov test. Figure 1 depicts the distributions of the three CPT outcome measures in the combined Haifa cohort to visually demonstrate that all three CPT outcomes deviate significantly from the normal 'bell-shaped' curve.

Analysis of the very large Tromsø study yielded the same conclusion. Table 2 shows that none of its CPT outcomes were normally distributed. Figure 2 depicts the distribution of cold pain tolerance and cold pain intensity. Among its 10,486 subjects, 7,157 (68.3\%) endured the entire $106 \mathrm{~s}$ immersion. The resulting frequency distribution is therefore severely right censored, as expected with an upper time limit cutoff. However, it is highly unlikely that extending this limit would significantly normalize the dataset, since pain ratings do not increase noticeably after the first 60 s, and for some participants they actually decrease (data not shown). 


\section{Efficacy of mathematical transformations for improving normality of CPT data}

Table 3 summarizes the results of our analysis of the usefulness of the two most common transformations used in attempt to normalize skewed datasets (logarithmic ( $\log$ ) and square root (SqR) transformations $)^{30}$ such as the skewed CPT data from the Haifa datasets depicted in Figure 1. For cold pain threshold, Log transformation normalized 50\% (4/8) of the datasets as assessed by the Shapiro-Wilk test but only $12.5 \%$ (1/8) of the datasets as assessed by the Kolmogorov-Smirnov test. The SqR transformation only normalized one $(12.5 \%)$ dataset (\#1) as measured by both Shapiro-Wilk and Kolmogorov-Smirnov tests. For cold-pain tolerance, Log transformation normalized 25\% (2/8) of the Haifa datasets as analyzed by the Shapiro-Wilk test, and 62.5\% (5/8) of datasets by the Kolmogorov-Smirnov test. The SqR transformation was unable to normalize any of the cold-pain tolerance datasets. For pain intensity, both the $\mathrm{Log}$ and the $\mathrm{SqR}$ transformations were able to normalize data from one data set (based only on the Kolmogorov-Smirnov test). Similarly, following Log and SQR transformations the Troms $\varnothing$ Study data remained non-normally distributed (Table 4). Overall, for both cold-threshold and the cold-tolerance data, the Troms $\varnothing$ data could be normalized $22 \%$ (7/32) of the time. Only 6\% (2/32) of the attempts to normalize cold-pain intensity data were successful.

\section{Monte Carlo simulations for comparing power of the Wilcoxon Mann Whitney (WMW) and the t-test}

Examples of the results of Monte Carlo simulations from the \#5 Haifa cohort are presented in Table 5. The left side of the table reports the power of the t-test, for 5 different effect sizes $(0.2,0.5,0.8,1.2, \& 2.0)$ assuming a treatment modeled as a shift in location parameter, with nominal a set at three different levels $(0.05,0.01$, and 0.001$)$. The right side of the table reports the power of WMW test under the same study conditions. For the entire spectrum of effect sizes, the WMW was found to be much more powerful than the t-test. The results in this table simulate balanced layouts of $n=60$ (30 participants in each group). Similar results, demonstrating the superiority of the WMW test for all other study conditions (48 tables per cohort) are not shown but can be requested from S. Sawilowsky.

Figure 3 summarizes the results of simulation to compare the average power of the t-test and the WMW across all 16 sample sizes studied with a set at 0.05 and evaluating power over the full range of effect sizes. This revealed that for all three CPT measures and for small $(0.2)$, medium (0.5) and large (0.8) effect sizes, the WMW was superior to the t-test. For example, for $\mathrm{a}=0.05$, using the pain threshold and an effect size of 0.2 , the power of the WMW (0.50) averaged 2.5 times larger than the power of the t-test $(0.21)$. For medium effect size, power of the WMW (0.78) was $18 \%$ higher than the power of the t-test $(0.66)$, while for large effect size the power of the WMW (0.97) was 9\% greater than for the t-test $(0.89)$. The only situation in which the power of the t-test approached that of the WMW was for huge effect sizes (1.2 and 2).

Similarly, for pain tolerance outcomes, $\mathrm{a}=0.05$, and simulation based on effect size of 0.2 , the average power of the WMW (0.50) was 2.5 times larger than the t-test $(0.20)$. For medium effects the WMW (0.87) had 36\% more power than the t-test (0.64), and for large 
effects the WMW (0.97) had 9\% greater than the t-test (0.89). The only situation in which the power of the t-test approached that of the WMW was for huge effect sizes (1.2 and 2).

For pain intensity outcomes and effect size of 0.2 , average power of the WMW (0.57) was almost three times larger than for the t-test (0.21). For medium effects, the power of the WMW (0.87) was 34\% higher than the t-test power (0.65), and for large effect size the WMW (0.98) was $10 \%$ more powerful than for the t-test (0.89). The WMW and t-test had similar power only for huge effect sizes (1.2 and 2), because above an effect size of 1.2 they both reach a maximum plateau at power of 1 . For $a=0.01$ and 0.001 , the superior power of the WMW was even greater as sample sizes increased.

To assess the possible role of sample size on the power of WMW and the t-test, the average power across all effect sizes was calculated and plotted (Figures 4) with sample size on the horizontal axis (a level=0.05). This demonstrates that the WMW is more powerful than the t-test for all simulated sample sizes.

We also examined the role of group allocation, by comparing simulation results of the balanced vs. unbalanced group allocations of the WMW and the t-test (Figure S1). For small effect sizes, unbalanced allocation result in a $0.1-0.2$ power loss in both tests.

Additional simulation studies, based on all other cohorts revealed similar or even higher power advantage. For example, when simulation $(n 1=30, n 2=30$, alpha=0.05) used data from the Troms $\varnothing$ cohort $(n=10,486)$, the WMW enjoyed an enormous power advantage of over five times the comparative statistical power than the t-test ( $\mathrm{t}$-test power $=0.1914$, WMW power $=0.9961)$ for the tolerance dataset, and twice as powerful ( $\mathrm{t}$-test power $=0.1944$, WMW power=0.3892) for the pain intensity dataset.

When simulating $\left(\mathrm{n}_{1}=30, \mathrm{n}_{2}=30\right.$, alpha=0.05) using data from Haifa smallest cohort (cohort $\# 1, \mathrm{n}=40$ ), the WMW conveyed a huge power advantage for tolerance (t-test power=0.1913, WMW power=0.8338) and intensity data (t-test power=0.1915, WMW power=0.3532).

However, there was only a slight benefit for data sampled from the threshold dataset (t-test power=0.1922, WMW power=0.1960), Similarly, when simulating $(\mathrm{n} 1=30, \mathrm{n} 2=30$, alpha $=0.05)$ using the biggest Haifa cohort $(\# 7, \mathrm{n}=193)$, analysis of the tolerance (t-test power $=0.2059$, WMW power=0.6613) and intensity data (t-test power=0.1928, WMW power $=0.3375$ ) data demonstrated large power advantages, whereas negligible differences was found for threshold data (t-test power=0.2135, WMW power=0.2000).

\section{Discussion}

These results show that although the vast majority (72\%) of published CPT studies used parametric statistics, only $39 \%$ even mentioned testing for normality, and among them, only $18 \%$ found cold-pain tolerance to be normally distributed. Pain researchers may not be fully aware of the implication of this and manuscript reviews may not sufficiently emphasize it. Secondly, our analysis of raw data from multiple independent small, medium, and very large CPT datasets reveals that CPT outcomes are only rarely normally distributed and most often mathematical transformations are ineffective in normalizing non-normal sets of CPT data. The results also suggest that when considering transformations of non-normal cold 
thresholds, Log transformation, followed by Shapiro-Wilk test for normality is preferable, whereas for cold tolerance data, Log transformation followed by Kolmogorov-Smirnov test is preferable. Other statistical tests may be better for other pain measures. For example, survival analysis may be preferable for tolerance data. ${ }^{26}$

Thirdly, Monte Carlo simulations based on multiple CPT datasets established that the nonparametric WMW test is almost always more statistically powerful than the t-test, meaning that applying t-tests to non-normal CPT data sets might compromise study conclusions and lead to false negative conclusions. The power superiority of non-parametric methods for non-normal data sets is most relevant for between-group comparisons when the treatment is modeled as a shift in location (along the $\mathrm{x}$ axis) of the data curve. In contrast, within-group comparisons (i.e. cross-over, a.k.a. before vs. after design) are more powerful to begin with (given the reduction in error variance associated with individual differences), thus nonparametric approaches offer little power advantage under these conditions. ${ }^{3}$ Among the reviewed studies, the most relevant to our discussion are those with between-subject-design, but $75 \%$ of these used parametric analyses. Taken together, these results suggest that parametric analyses should not be the primary statistical method for analyzing CPT data as they currently are. As previously reported,, several common misconceptions may lead researchers to choose parametric approaches when non-parametric statistics are more appropriate. ${ }^{22}$ False statements are common, such as "Wilcoxon should only be used when the data is originally in the form of ranks", or "in small samples studies", or that "that is more powerful".

The greater power of nonparametric tests for between-group studies means that using them can reduce the number of subjects needed for adequate power. For instance, our pain tolerance data show that when comparing treatment and control groups containing 30 subjects each, the power of the WMW test is 0.3195 for detecting a typical small $(0.20)$ treatment effect, whereas analysis by t-test would require 120 subjects per group for comparable power (.3401). It is commonly stated that the WMW test should be used only for small samples, but Sawilowsky refuted this assumption. ${ }^{22}$ For instance, in analyzing cold-pain intensity, if $a=0.001$, effect size $=0.2$, and sample size $=240$ per group, the onetailed power for the WMW was .92 whereas power of the t-test was only 0.14 . To achieve similar power for t-test analysis, group size would have to be 1,011. Thus, nonparametric statistics can save time, effort, risk to subjects, and cost.

Strengths of our study include the fact that we analyzed multiple independent datasets from different countries, covering the full range of sample sizes used for CPT pain research ( $\mathrm{n}=$ 40 - 10,468). Importantly, although our 7 Haifa cohorts included only healthy young students, our findings can be generalized to the general population since the very large Troms $\varnothing$ study sampled unscreened healthy and unhealthy participant alike who are physically able to make it to the study site. $32 \%$ of the Troms $\varnothing$ cohort reported having chronic pain, a prevalence not elevated from that in the normal population in Norway ${ }^{1}$ or the U.S. ${ }^{8}$

Our Monte Carlo simulations also spanned the full gamut of sub-sample sizes $(40-480)$ as well as a wide range of group allocation scenarios. Group allocation is important because if 
poor results are found under balanced layout, this will be exacerbated for unbalanced layouts. Another strength is that we applied multiple methods (review of published studies, data re-analyses, and Monte Carlo simulation) all of which supported the conclusions that non-parametric statistics should be used to analyze CPT data.

A limitation is that we only analyzed CPT, one specific type of pain dataset. However, our results may have clinical implications because CPT is increasingly being used as a clinical tool due to the growing emphasis on mechanism-based pain diagnosis and the use of quantitative sensory testing. In addition, we propose that our conclusions likely apply to other types of human experimental pain research because many are designed with safety limits or artificial cut points that produce skewed distributions with one tail truncated. Measuring pain intensity using strong nociceptive stimuli also generates a skewed distribution with mostly high values and a truncated right tail and weak stimuli similarly produce truncated left tails. Only stimuli that elicit midrange responses avoid this. Findings from other scientific fields conclusively demonstrate the superiority of non-parametric approaches when the normality assumption is violated for treatments inducing shifts in location along the $\mathrm{x}$ axis of the dependent variable. ${ }^{16,19,20,25}$

These results are relevant for clinical trials of new pain treatments which often compare outcomes between two groups (treatment vs. control), that are likely to be non normally distributed. Most trials of pain therapies only include patients with pain intensities $\geq 4 / 10,{ }^{31}$ increasing the probability of non-normal distribution. Moreover, changes in pain intensity are commonly used as outcomes. Based on the fact that only some patients respond, ${ }^{7,8,32}$ skewed distributions can be expected. From our experience, patient reported outcome (PRO) data collected from studies of medical conditions is only rarely completely normal. Although they are often analyzed with parametric statistics, they can equally well be analyzed using non-parametric statistics which require fewer assumptions about the structure of the data and are equally efficient in most real data circumstance. "Since at the design stage of a clinical study we cannot be certain of the distribution of the outcome data, it seems prudent to assume non normality and design the analyses accordingly.

Another limitation is that we only compared the WMW and t-test, thus we cannot generalize to all parametric vs. non-parametric analyses. We know of only two prior pain-related studies, one comparing the power of WMW vs. analysis of covariance (ANCOVA) in a simulation based on headache and shoulder-pain trials. ${ }^{33}$ Post-treatment scores were compared between groups using baseline score as covariant for ANCOVA. This demonstrated that ANCOVA is better for moderate but not for extremely non-normal distributions, but as Sawilowky noted, there are no good nonparametric equivalents to ANCOVA. ${ }^{24}$ Torrence et al. ${ }^{27}$ compared the power of ANOVA, t-tests, Kruskall-Wallis, Mann-Whitney U-tests, and evaluated bootstrapping and Log transformation for analyzing skewed results of SF-36 questionnaire (the Medical Outcomes Study 36 Item Short-Form), a very common secondary outcome measure in pain trials. ${ }^{2}$ They concluded that the statistical tests were equivalent, but they only studied a very large cohort $(\mathrm{n} \sim 3,000)$ with very high power, thus their conclusions cannot be applied to studies of hundreds or tens of subjects, or when small effect sizes are anticipated. 
Another limitation deserves notice: Given the large number of subjects pooled in the current study, assessing relations between demographic characteristics and pain measures distribution is possible, but it is beyond the scope of the current paper.

Current approaches to evaluating normality of datasets include the classical Lilliefor's test, (an adjustment to the Kolmogorov-Smirnov test), the Shapiro-Wilk's test, the AndersonDarling test, and the D'Agostino-Pearson test. An alternative is visual inspection of plotting, such as a Q-Q plot. However both approaches constitute multiple comparisons and thus increase the probability of Type I error and require corrections such as Bonferroni's. ${ }^{21}$ Therefore, if the data are not known to be normally distributed, or suspected to be nonnormally distributed, then the parametric test should be avoided.

Our results suggest that the current automatic application of parametric analyses to CPT studies should change, and require demonstrating the normality of outcome data. For the vast majority of CPT studies with non-normal data, the nonparametric Wilcoxon Mann Whitney test is much more powerful than the parametric t-test. Although statistical textbooks recommend nonparametric analyses for non-normally distributed data, and we have demonstrated that normal distributions are rare in CPT data as in biomedical research in general, ${ }^{10,14,22}$ parametric analysis is still routinely used CPT studies.

\section{Supplementary Material}

Refer to Web version on PubMed Central for supplementary material.

\section{Acknowledgments}

Supported in part by the Public Health Service (NINDS K24-NS059892). We thank Suzan Erica MSc, Aviram Shuki MA, Demeter Naor MA, Yael Golan-Vered MA, from the University of Haifa, for their help with data collection.

\section{Reference List}

1. Breivik H, Collett B, Ventafridda V, Cohen R, Gallacher D. Survey of chronic pain in Europe: prevalence, impact on daily life, and treatment. Eur J Pain. 2006; 4:287-333. [PubMed: 16095934]

2. Bradley JV. Robustness? British Journal of Mathematical and Statistical Psychology. 1978; 31:14452.

3. Bridge PD, Sawilowsky SS. Increasing physicians' awareness of the impact of statistics on research outcomes: comparative power of the t-test and Wilcoxon Rank-Sum test in small samples applied research. J Clin Epidemiol. 1999; 52:229-35. [PubMed: 10210240]

4. Cohen, J. Statistical power analysis for the behavioral sciences. 2. Hillsdale, NJ: Erlbaum; 1988.

5. Colliver JA, Manchikanti L, Markwell SJ. Evaluation and comparison of the distributions of gastric $\mathrm{pH}$ and hydrogen ion concentration. Anesthesiology. 1987; 67:391-4. [PubMed: 3631613]

6. Dworkin RH, Turk DC, Katz NP, Rowbotham MC, Peirce-Sandner S, Cerny I, Clingman CS, Eloff BC, Farrar JT, Kamp C, McDermott MP, Rappaport BA, Sanhai WR. Evidence-based clinical trial design for chronic pain pharmacotherapy: a blueprint for ACTION. Pain. 2011; 152:S107-S115. [PubMed: 21145657]

7. Harden N, Cohen M. Unmet needs in the management of neuropathic pain. J Pain Symptom Manage. 2003; 25:S12-S17. [PubMed: 12694988]

8. Institute of Medicine (US) Committee on Advancing Pain Research, Care, and Education. Relieving Pain in America: A Blueprint for Transforming Prevention, Care, Education, and Research. Washington (DC): National Academies Press (US); 2011. 
9. Jain KK. Current challenges and future prospects in management of neuropathic pain. Expert Rev Neurother. 2008; 8:1743-56. [PubMed: 18986244]

10. Bradley, James V. A Common Situation Conducive to Bizarre Distribution Shapes A Common Situation Conducive to Bizarre Distribution Shapes. The American Statistician. 1977; 31:147-50.

11. Johansen A, Schirmer H, Stubhaug A, Nielsen CS. Persistent post-surgical pain and experimental pain sensitivity in the Tromso study: comorbid pain matters. Pain. 2014; 155:341-8. [PubMed: 24145207]

12. Koenig J, Jarczok MN, Ellis RJ, Bach C, Thayer JF, Hillecke TK. Two-week test-retest stability of the cold pressor task procedure at two different temperatures as a measure of pain threshold and tolerance. Pain Pract. 2014; 14:E126-E135. [PubMed: 24256148]

13. Manly, BFJ. Randomization, Bootstrap and Monte Carlo Methods in Biology. 2. Boca Raton, FL: Chapman-Hall/CRC; 1997.

14. Micceri T. The Unicorn, The Normal Curve, and Other Improbable Creatures. Psychological Bulletin. 1989; 105:156-66.

15. Modir JG, Wallace MS. Human experimental pain models 2: The cold pressor model. Methods Mol Biol. 2010; 617:165-8. [PubMed: 20336421]

16. Moran JL, Solomon P. Worrying about normality. Critical Care and Resuscitation. 2002; 4:316-9. [PubMed: 16573445]

17. Pud D, Eisenberg E, Sprecher E, Rogowski Z, Yarnitsky D. The tridimensional personality theory and pain: harm avoidance and reward dependence traits correlate with pain perception in healthy volunteers. Eur J Pain. 2004; 8:31-8. [PubMed: 14690672]

18. Pud D, Golan Y, Pesta R. Hand dominancy--a feature affecting sensitivity to pain. Neurosci Lett. 2009; 467:237-40. [PubMed: 19853018]

19. Sawilowsky SS. Comments on using alternatives to normal theory statistics in social and behavioral science. Canadian Psychology. 1993; 34:398-406.

20. Sawilowsky SS. Comments on using robust statistics in social and behavioral science. British Journal of Mathematical and Statistical Psychology. 1998; 51:49-52.

21. Sawilowsky SS. Fermat, Schubert, Einstein, and Behrens-Fisher: The probable difference between two means when $\sigma 12 \approx 22$. Journal of Modern Applied Statistical Methods. 2002; 1(2):461-472.

22. Sawilowsky SS. Misconceptions Leading to Choosing the t Test Over the Wilcoxon MannWhitney Test for Shift in Location Parameter. Journal of Modern Applied Statistical Methods. 2005; 4:598-600.

23. Sawilowsky SS. New effect size rules of thumb. Journal of Modern Applied Statistical Methods. 2009; 8:597-9.

24. Sawilowsky SS. Nonparametric tests of interaction in experimental design. Review of Educational Research. 1990; 60:91-126.

25. Sawilowsky SS, Blair RC. A more realistic look at the robustness and type II error properties of the $t$ test to departures from population normality. Psychological Bulletin. 1992; 111:353-60.

26. Stabell N, Stubhaug A, Flaegstad T, Nielsen CS. Increased pain sensitivity among adults reporting irritable bowel syndrome symptoms in a large population-based study. Pain. 2013; 154:385-92. [PubMed: 23320954]

27. Torrance N, Smith BH, Lee AJ, Aucott L, Cardy A, Bennett MI. Analysing the SF-36 in population-based research. A comparison of methods of statistical approaches using chronic pain as an example. J Eval Clin Pract. 2009; 15:328-34. [PubMed: 19335493]

28. Treister R, Pud D, Ebstein RP, Eisenberg E. Dopamine transporter genotype dependent effects of apomorphine on cold pain tolerance in healthy volunteers. PLoS One. 2013; 8:e63808. [PubMed: 23704939]

29. Treister R, Pud D, Ebstein RP, Laiba E, Gershon E, Haddad M, Eisenberg E. Associations between polymorphisms in dopamine neurotransmitter pathway genes and pain response in healthy humans. Pain. 2009; 147:187-93. [PubMed: 19796878]

30. Tukey, JW. Exploratory Data Analysis. 1. Massachusetts: Addison-Wesley; 1977.

31. 
32. Turk DC, Dworkin RH, Burke LB, Gershon R, Rothman M, Scott J, Allen RR, Atkinson JH, Chandler J, Cleeland C, Cowan P, Dimitrova R, Dionne R, Farrar JT, Haythornthwaite JA, Hertz S, Jadad AR, Jensen MP, Kellstein D, Kerns RD, Manning DC, Martin S, Max MB, McDermott MP, McGrath P, Moulin DE, Nurmikko T, Quessy S, Raja S, Rappaport BA, Rauschkolb C, Robinson JP, Royal MA, Simon L, Stauffer JW, Stucki G, Tollett J, von Stein T, Wallace MS, Wernicke J, White RE, Williams AC, Witter J, Wyrwich KW. Initiative on Methods, Measurement and Pain Assessment in Clinical Trials: Developing patient-reported outcome measures for pain clinical trials: IMMPACT recommendations. Pain. 2006; 125:208-15. [PubMed: 17069973]

33. Vadalouca A, Siafaka I, Argyra E, Vrachnou E, Moka E. Therapeutic management of chronic neuropathic pain: an examination of pharmacologic treatment. Ann N Y Acad Sci. 2006; 1088:164-86. [PubMed: 17192564]

34. Vickers AJ. Parametric versus non-parametric statistics in the analysis of randomized trials with non-normally distributed data. BMC Med Res Methodol. 2005; 5:35. [PubMed: 16269081]

35. von Baeyer CL, Piira T, Chambers CT, Trapanotto M, Zeltzer LK. Guidelines for the cold pressor task as an experimental pain stimulus for use with children. J Pain. 2005; 6:218-27. [PubMed: 15820909] 


\section{Perspective}

These results demonstrate that parametric analyses of CPT data are routine but incorrect, and that they likely increase chances of failing-to-detect significant between-group differences. They suggest that non-parametric analyses become standard for CPT studies, and that assumptions of normality be routinely tested for other types of pain outcomes as well. 


\section{Highlights}

- The appropriateness of using parametric analysis on cold pressor data was studied

- In most previously published studies data distribution was not mentioned

- However, vast majority of these studies used parametric analyses

- CPT measures collected at 8 independent studies were not normally distributed

- Monte Carlo simulations reveal that non-parametric approach is more appropriate 


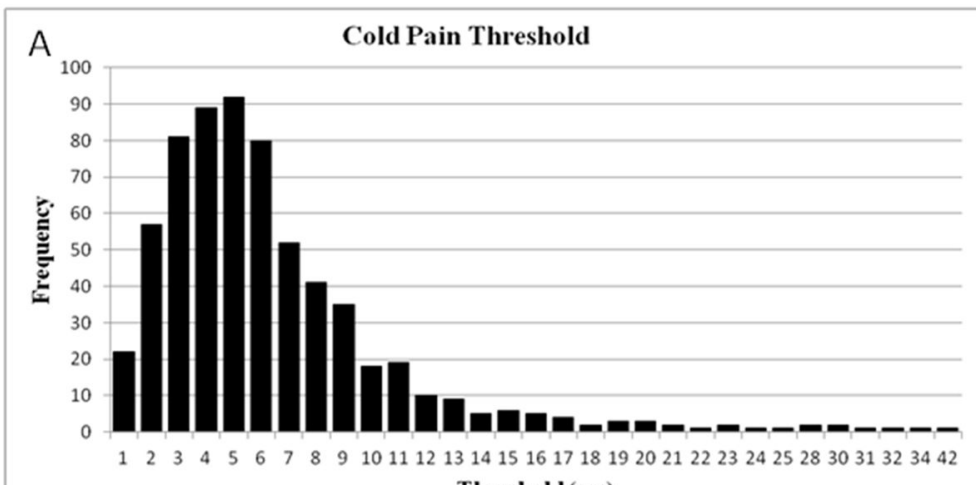
Threshold (sec)

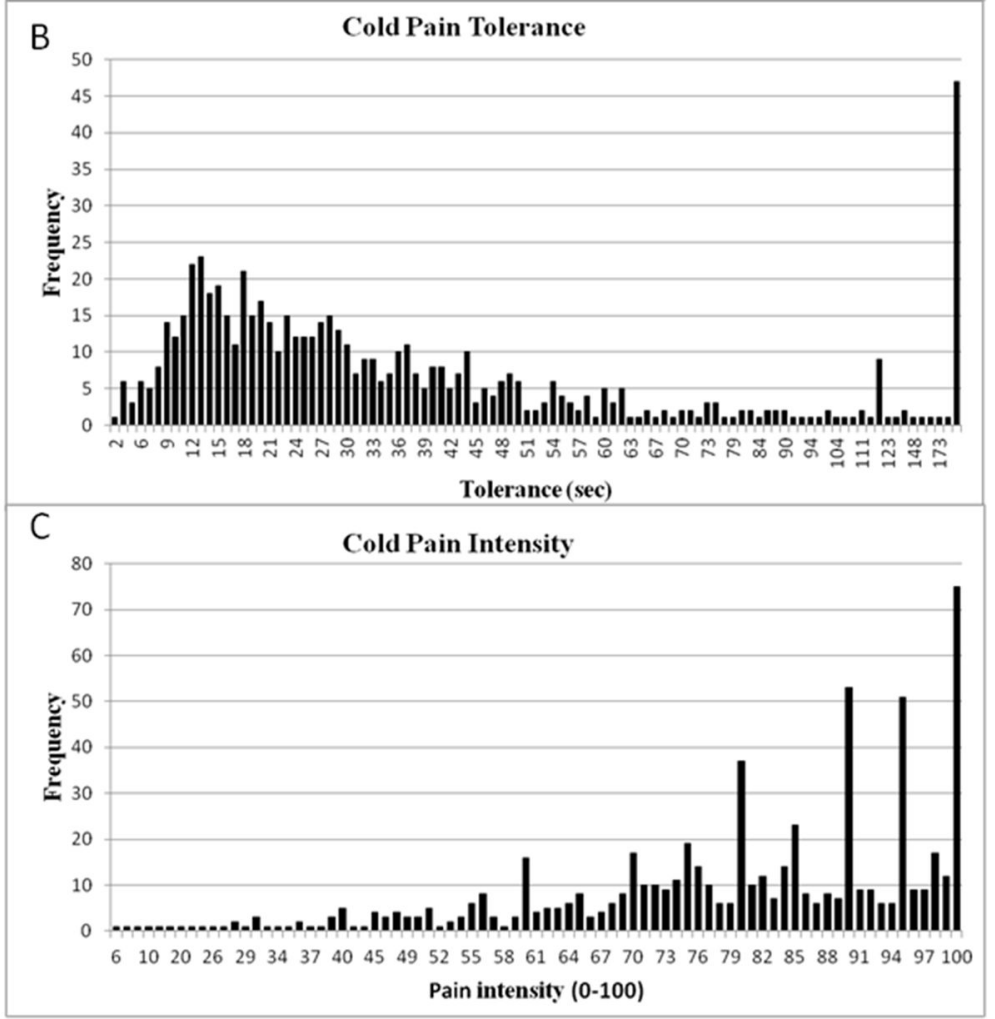

Figure 1.

Distribution of (A) cold-pain threshold, (B) cold-pain tolerance and (C) cold-pain intensity in the combined Haifa cohort $(n=648)$. 

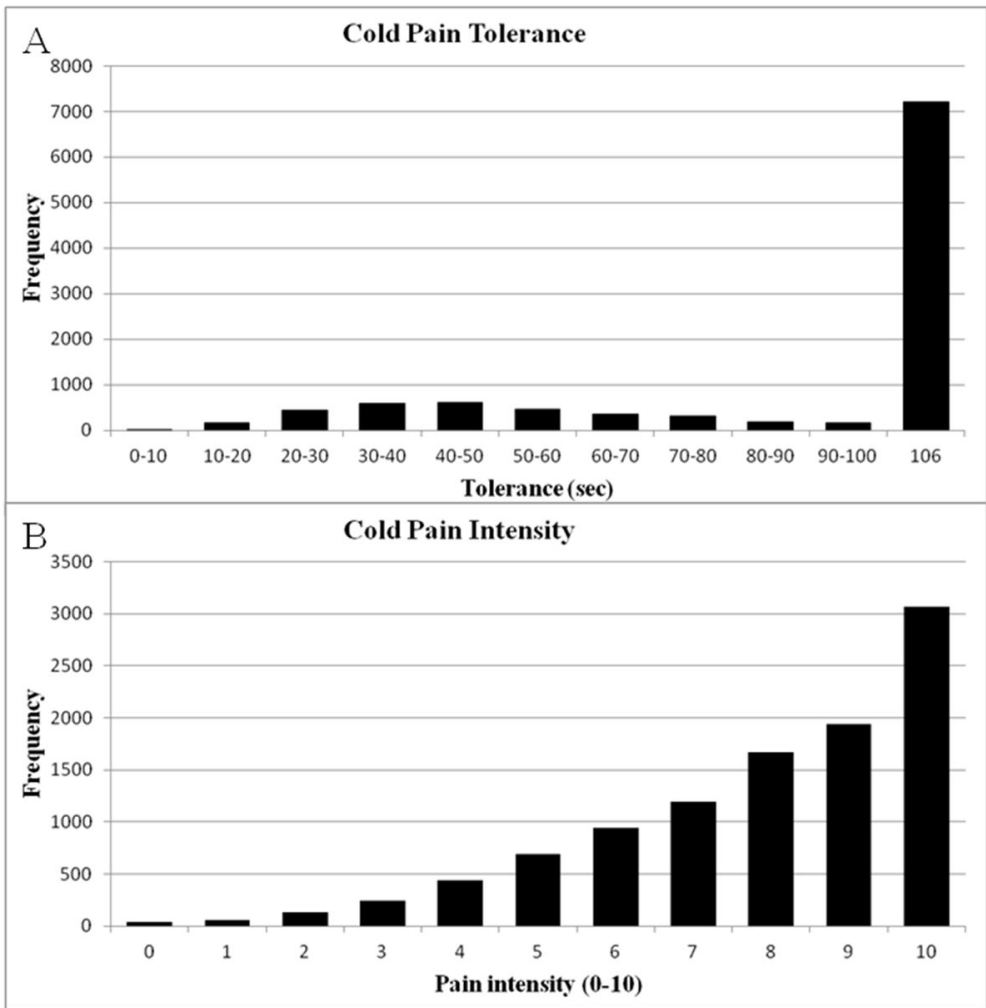

Figure 2.

Distribution of (A) cold-pain tolerance and (B) cold-pain intensity in the Troms $\varnothing$ Study cohort. 


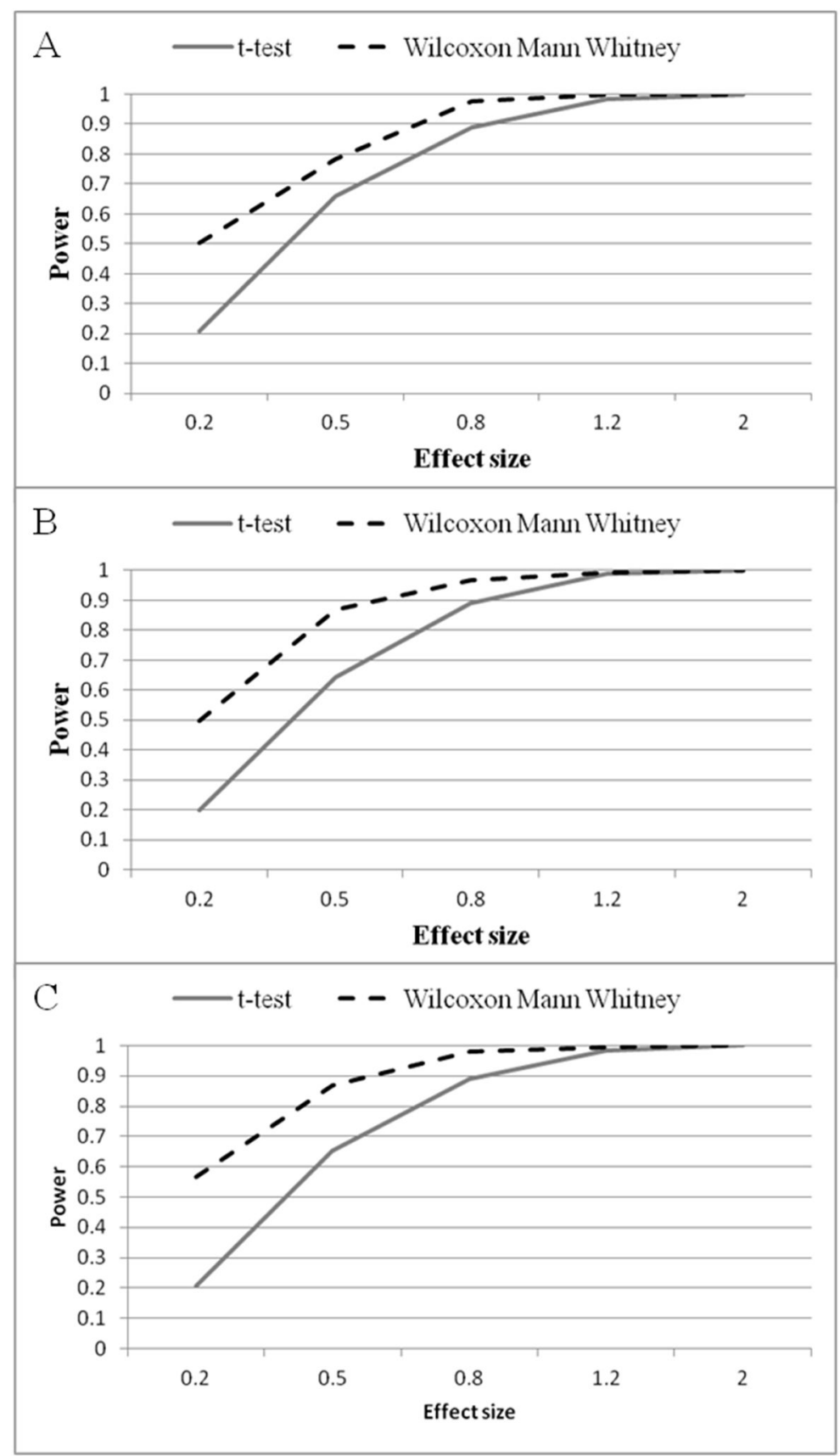

Figure 3.

Average power of the Independent Samples t-test and the Wilcoxon Mann Whitney across all effect size scenarios (a level=0.05). (A) For cold threshold scores (B) For cold tolerance scores; (C) For pain intensity scores. 


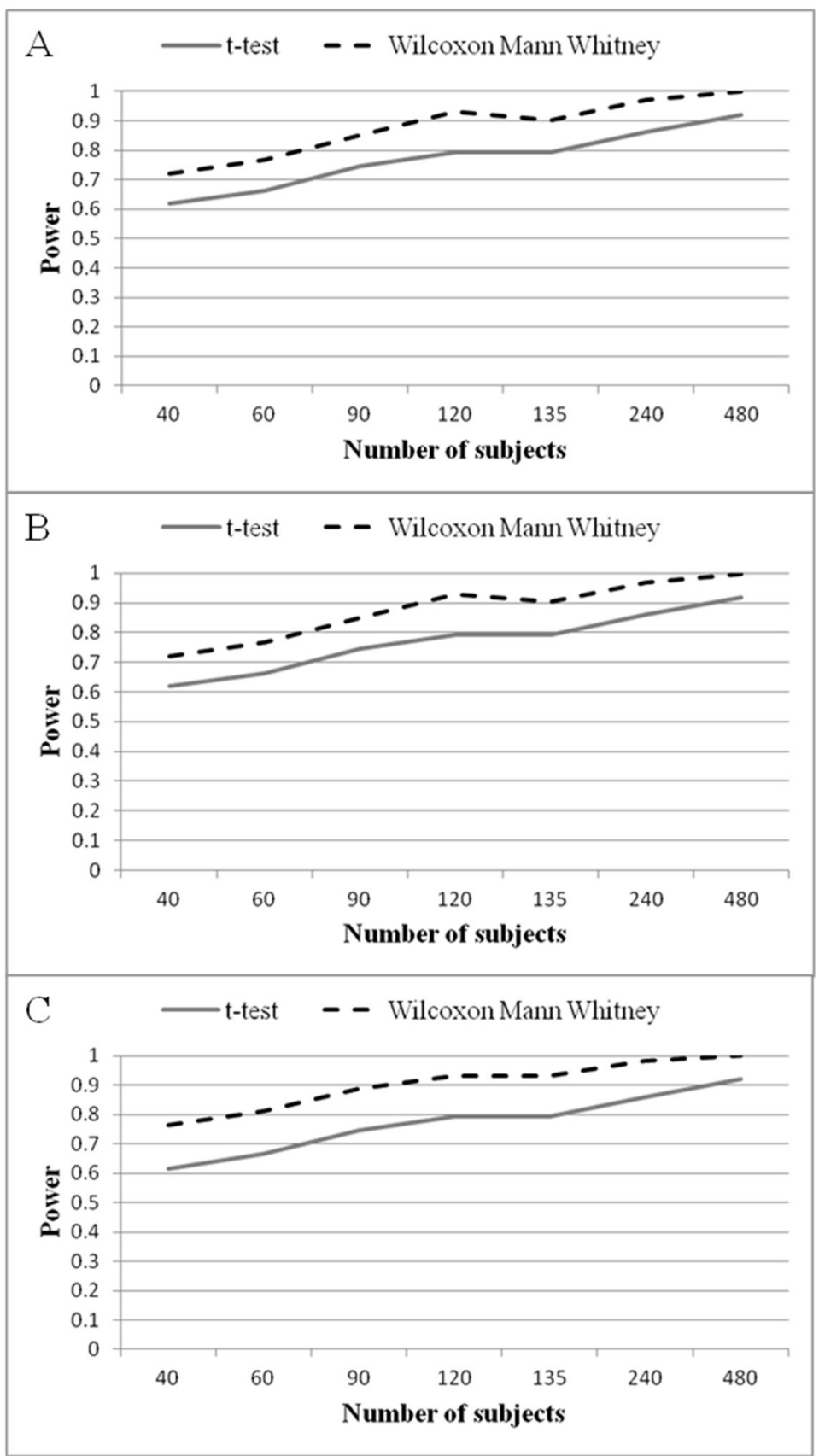

Figure 4.

Average power of the Independent Samples t-test and the Wilcoxon Mann Whitney across all number of subjects scenarios (a level=0.05). (A) Cold threshold scores (B) Cold tolerance scores; (C) Pain intensity scores. 


\section{Table 2}

Statistics of cold pain tolerance and pain intensity in the large population-based study cohort from Troms $\emptyset$.

\begin{tabular}{llc}
\hline & The Tromsø cohort & \\
\hline $\mathrm{n}$ & & 10,486 \\
\% Female & & 0.515 \\
Age range & & $30-87$ \\
\hline Tolerance & Mean & 88.17 \\
& Mode & 106 \\
& Median & 106 \\
& Range & $5.4-106$ \\
& Skewness, Kurtosis & $-1.26,-0.39$ \\
& SW test (P-value) & $\mathrm{P}<0.001$ \\
& KS test (P-value) & $\mathrm{P}<0.001$ \\
\hline Pain Intensity & Mean & 7.84 \\
& Mode & 10 \\
& Median & $0-10$ \\
& Range & $-1.01,0.49$ \\
& Skewness, Kurtosis & $\mathrm{P}<0.001$ \\
& SW test (P-value) & \\
& KS test (P-value) & $\mathrm{P}<0.001$ \\
\hline
\end{tabular}

J Pain. Author manuscript; available in PMC 2016 June 01. 


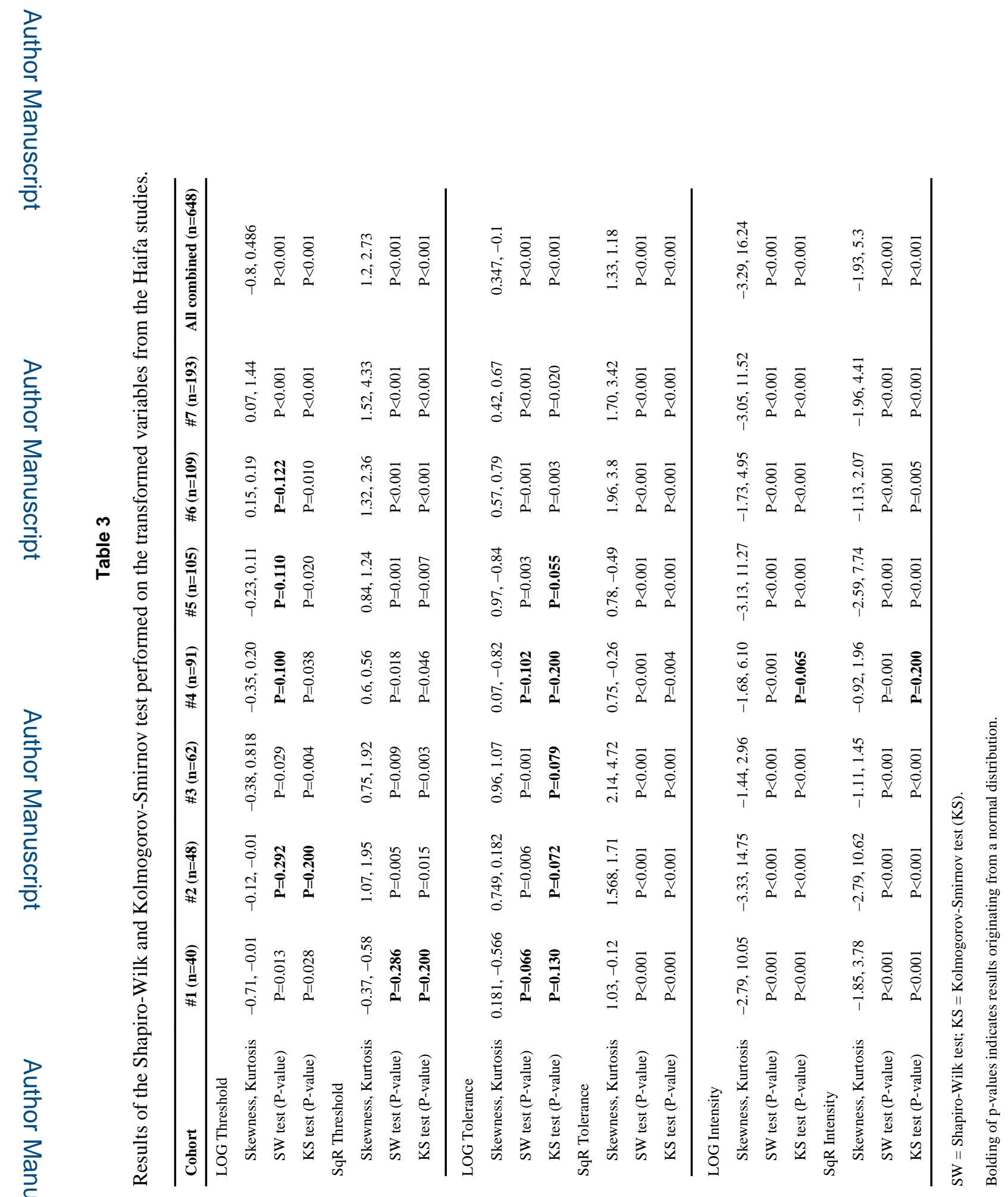




\section{Table 4}

Results of the Shapiro-Wilk and Kolmogorov-Smirnov test performed on the transformed variables from the Tromsø study.

\begin{tabular}{lc}
\hline The Tromsø cohort & \\
\hline LOG Tolerance & \\
Skewness, Kurtosis & $-1.81,2.697$ \\
SW test (P-value) & $\mathrm{P}<0.001$ \\
KS test (P-value) & $\mathrm{P}<0.001$ \\
SqR Tolerance & \\
Skewness, Kurtosis & $-1.45,0.75$ \\
SW test (P-value) & $\mathrm{P}<0.001$ \\
KS test (P-value) & $\mathrm{P}<0.001$ \\
\hline LOG Intensity & \\
Skewness, Kurtosis & $-2.16,6.42$ \\
SW test (P-value) & $\mathrm{P}<0.001$ \\
KS test (P-value) & $\mathrm{P}<0.001$ \\
SqR Intensity & \\
Skewness, Kurtosis & $-1.89,5.66$ \\
SW test (P-value) & $\mathrm{P}<0.001$ \\
KS test (P-value) & $\mathrm{P}<0.001$ \\
\hline
\end{tabular}

$\mathrm{SW}=$ Shapiro-Wilk test KS $=$ Kolmogorov-Smirnov test $(\mathrm{KS})$. 


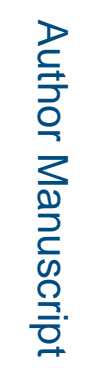

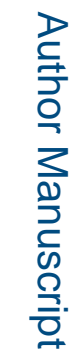

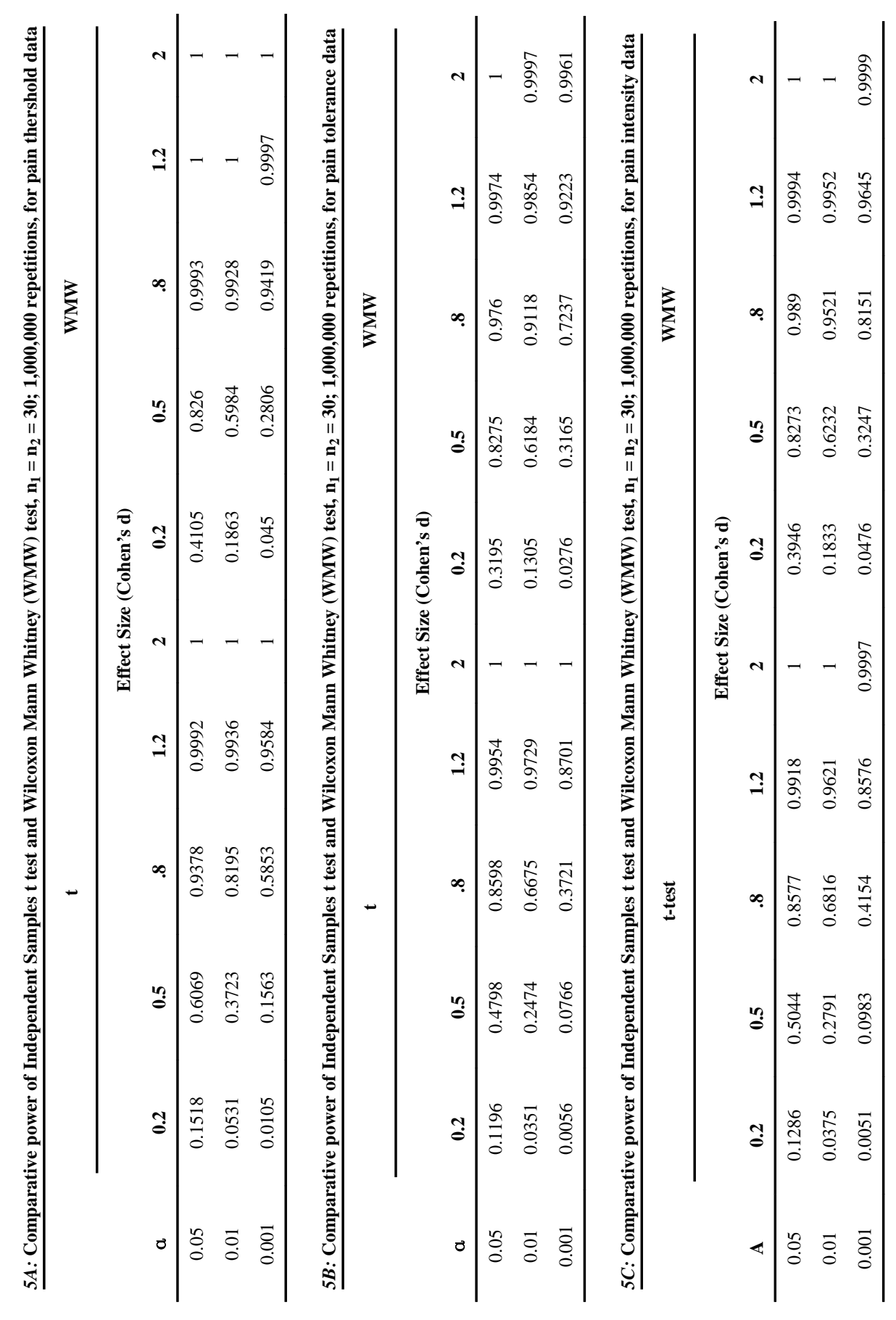

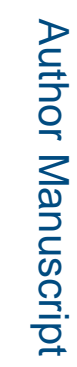

